\title{
An Introduction to the \\ Mathematical Theory of Vibrations of Elastic Plates
}


This page is intentionally left blank 


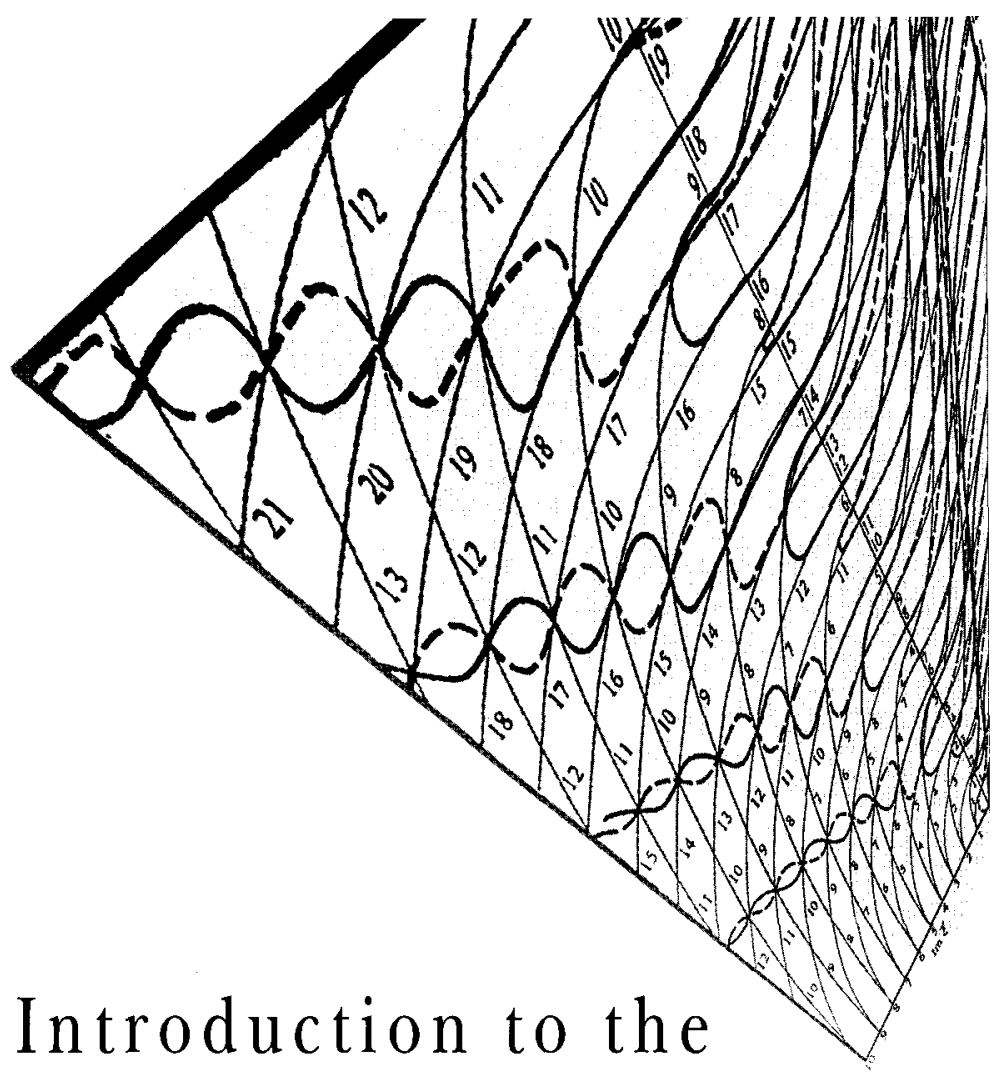

\title{
Mathematical Theory of
}

Vibrations of Elastic Plates

\author{
R D Mindlin
}

Columbia University, USA

edited by Jiashi Yang

University of Nebraska-Lincoln, USA

\section{No world Scientific}




\section{Published by}

World Scientific Publishing Co. Pte. Ltd.

5 Toh Tuck Link, Singapore 596224

USA office: 27 Warren Street, Suite 401-402, Hackensack, NJ 07601

UK office: 57 Shelton Street, Covent Garden, London WC2H 9HE

\section{British Library Cataloguing-in-Publication Data}

A catalogue record for this book is available from the British Library.

\section{INTRODUCTION TO THE MATHEMATICAL THEORY OF VIBRATIONS OF ELASTIC PLATES}

Copyright $\odot 2006$ by World Scientific Publishing Co. Pte. Ltd.

All rights reserved. This book, or parts thereof, may not be reproduced in any form or by any means, electronic or mechanical, including photocopying, recording or any information storage and retrieval system now known or to be invented, without written permission from the Publisher.

For photocopying of material in this volume, please pay a copying fee through the Copyright Clearance Center, Inc., 222 Rosewood Drive, Danvers, MA 01923, USA. In this case permission to photocopy is not required from the publisher.

ISBN $981-270-381-0$ 


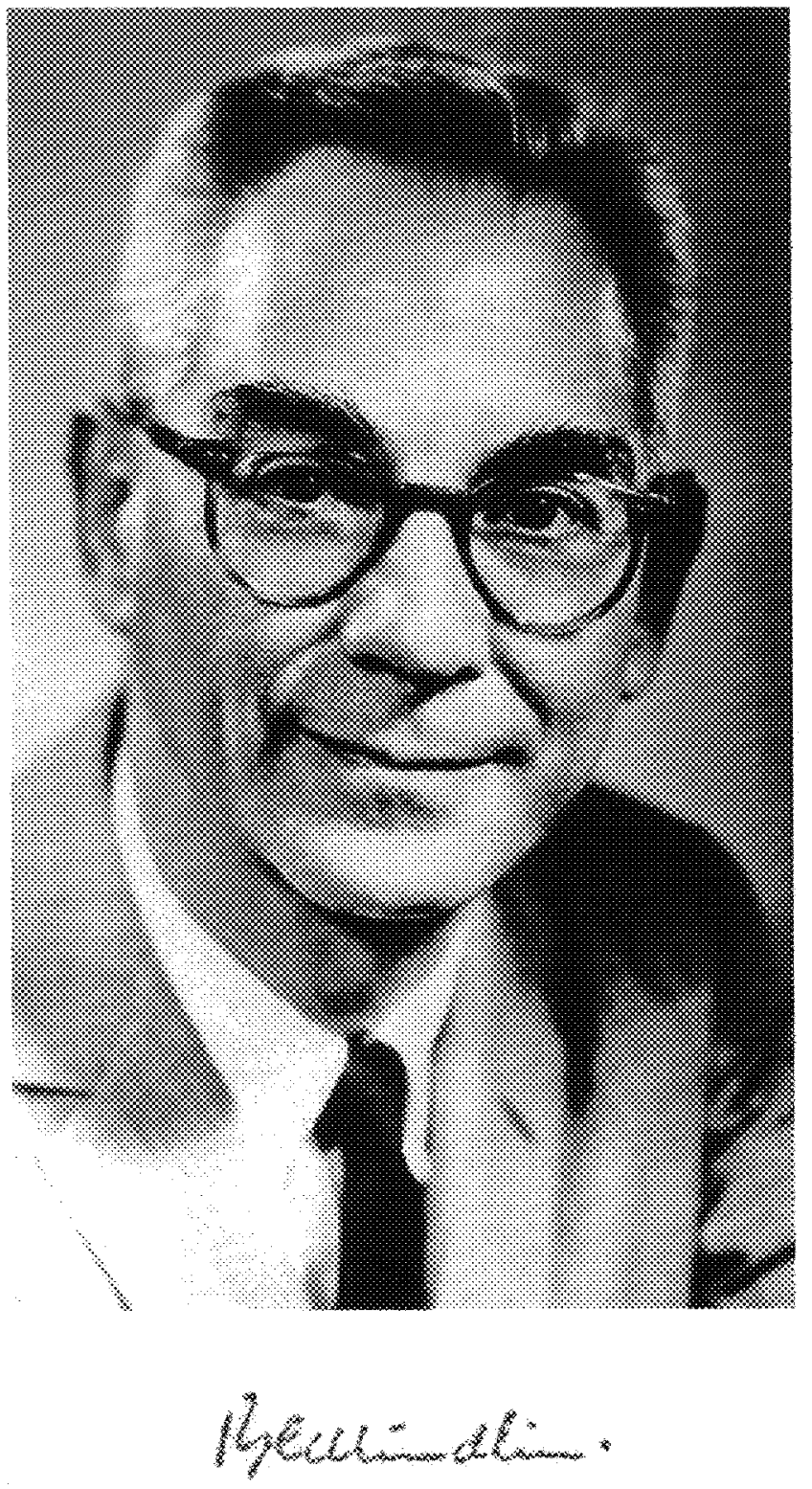

Raymond D. Mindlin (1906-1987) 
This page is intentionally left blank 


\section{Foreword}

2006 is the one hundredth anniversary of the birth of the late Professor Raymond D. Mindlin of Columbia University. The formal publication of this book based on a 1955 monograph [1] by Mindlin is my personal effort for this occasion.

The book systematically derives the two-dimensional theories of anisotropic elastic plates from the variational formulation of the three-dimensional theory of elasticity by power series expansions in the plate thickness coordinate. The uniqueness of solutions of the two-dimensional equations is also examined from the variational view point. The accuracy of the two-dimensional equations is judged by comparisons between the dispersion relations of the specific waves that the two-dimensional theories are supposed to describe with the prediction by the three-dimensional theory in the frequency-wave number range of interest.

The book mainly discusses dynamic problems. In addition to the so-called low-frequency waves like extension and flexure useful in traditional structural engineering, the book also discusses high-frequency waves including thickness-shear and thickness-stretch, which is rather unique and provides the theoretical foundation for acoustic wave devices made from anisotropic crystal plates. There are many aspects of plate theories that Mindlin knew more than anybody else then and now. The book has been a major reference for researchers of acoustic wave devices for half a century and will remain so in any foreseeable future. It is one of the classical works in mechanics that will long last. 
The Kane-Mindlin theory in the book for coupled extension and thickness-stretch motions of plates was later found to be flawed. It needs to be read with a later paper [2] for coupled extension, thickness-stretch, and symmetric thickness-shear (the Mindlin-Medick theory).

I would like to thank Ms. Liu He of the Central South University in Changsha, Hunan, China for typing a major portion of the manuscript, and Miss Liang Gu, a student of CSU, for typing the rest of it. Miss Xiang Cui, a graduate student of Southwest University in China, proofread the first draft under partial support from the Institute of Mechanics and Sensing Technologies of CSU. The figures in this book were directly scanned from [1] which was believed to be prepared entirely by Mindlin himself. A list for errors in the original manuscript and their corrections [3], prepared by one of Mindlin's students, my $\mathrm{Ph}$. D. advisor, Professor Peter C. Y. Lee of Princeton University, has been partially taken into consideration in the preparation of this book. The biographical sketch of Mindlin and his students' list are based on [4]. More information about Mindlin and his contributions can be found in $[5,6]$. The index was prepared by myself as the editor of the book.

September 2006

Jiashi Yang

Lincoln, Nebraska

[1] R. D. Mindlin, An Introduction to the Mathematical Theory of Vibrations of Elastic Plates, A monograph prepared for U. S. Army Signal Corps Engineering Laboratories, Fort Monmouth, N. J., Department of the Army Project 3-99-11-022, Signal Corps Project 142B, Signal Corps Contract DA-36-039 SC-56772, 1955.

[2] R. D. Mindlin and M. A. Medick, Extensional vibrations of elastic plates, ASME J. Appl. Mech., 26, 561-569, 1959. 
[3] P. C. Y. Lee, Errata, http://www.ieee-uffc.org/archive/uffc/books/books/ bk11/bk11000f.pdf.

[4] Wikipedia, The Free Encyclopedia, Bibliographic details for Raymond D. Mindlin, $\mathrm{http}$ ://en.wikipedia.org/w/index.php?title= Raymond_D._Mindlin\&oldid=59827080.

[5] R.D. Mindlin and Applied Mechanics, edited by George Herrmann, Pergamon Press, 1974.

[6] The Collected Papers of Raymond D. Mindlin, edited by H. Deresiewicz, M.P. Bieniek, F.L. DiMaggio, Springer-Verlag, 1989. 
This page is intentionally left blank 


\section{Preface}

The laws which govern the small vibrations of elastic plates are contained in the three-dimensional equations of the linear theory of elasticity. There is no essential difficulty in obtaining solutions of the equations for infinite plates, at least as far as the establishment of the secular equations. When the faces of the plate are free of traction, the solutions reveal the existence of an infinite number of modes of vibration for each wave-length in the plane of the plate. In a finite plate, with free or clamped edges, each of these modes (or its overtones) couples with all the others (or their overtones), leading to an extraordinarily complex spectrum of frequencies whose details are buried in an infinite system of transcendental equations.

Inasmuch as the governing differential equations are linear, each of the modes of an infinite plate has overtones in a finite plate, but no subtones. Accordingly, the lower modes of an infinite plate influence the high-frequency spectrum of a finite plate but the high modes of an infinite plate do not greatly affect the spectrum of a finite plate at lower frequencies. This circumstance permits the formulation of approximate equations with high-frequency cut-offs at various levels. The best known examples are the classical, two-dimensional equations of vibration of thin plates. The Germain-Lagrange-Cauchy flexural equations and the Poisson-Cauchy extensional equations contain only the lowest modes of an infinite plate and describe adequately the frequency spectra of finite plates as long as the frequency is well below that of the lowest absent mode. 
The extension of the two-dimensional equations to accommodate higher frequencies involves the incorporation of the next higher modes of an infinite plate. The first steps in this direction have led to applications (see the Appendix) of interest in the field of frequency control. As a result, an advisory committee, composed of E. A. Gerber, R. A. Sykes and K. S. Van Dyke, recommended to the Office of the Chief Signal Officer that a monograph be prepared in which the derivation of the new equations might be explained in detail.

It turned out that a reasonably full exposition of the foundations of the theory of vibrations of plates required preliminary studies in areas hitherto unexplored. These investigations have proved so fruitful and so necessary to an understanding of the theory that they comprise the major part of the monograph. The equations which formed the basis of the previous applications now appear as almost trivial special cases of more general equations covering a much wider field of applications.

Two-dimensional equations are, of course, approximations. The first question that arises in attempting to explain the development of the equations is "What is it that is being approximated?" The point of view adopted here is that the relation between frequency and wave-length is the element of prime importance. Accordingly, it is the aim to produce equations which will yield the appropriate relation, at least in a limited range. Now, the appropriate relation is the one given by the three-dimensional equations and, if these could be solved for all shapes and boundary conditions, there would be no need for approximations. Fortunately, it is only essential to have available the solution for an infinite plate. For the isotropic material this was given by Rayleigh in 1889.

Most of Chapter 2 is devoted to the study of Rayleigh's problem. Of particular interest is the method adopted to lead up to the final solution via the simpler problem of mixed boundary conditions. This enables the 
prediction of some of the major features of the complicated, coupled spectrum without detailed analysis or computations. Rayleigh's final transcendental equations are deceivingly simple in appearance. After more than sixty years during which the roots in various regions had been studied extensively (notably by Lamb in 1917 and Holden in 1951) there still remained unexplored the part of the spectrum of greatest importance for the development of two-dimensional equations. This part (the high-frequency, long wave-length region) is analyzed in detail in Section 2.11 .

Analogous to Rayleigh's solution, there is one by Ekstein (in 1945) for the monoclinic crystal, which has important applications to the rotated Y-cuts of quartz. In view of the length of time it has taken to gain an understanding of Rayleigh's problem it is not surprising that Ekstein's solution of a much more complicated problem has not yet been explored fully. Inasmuch as a large portion of the monograph is devoted to crystal plates, it would have been appropriate to describe Ekstein's problem in as great detail as Rayleigh's. However, there was not enough time available to complete the work. Consequently, a test of the first-order approximation for crystals, analogous to the test of the equations for isotropic plates at the end of Chapter 5, had to be postponed.

As early as 1828 , Poisson and Cauchy had already deduced the two-dimensional equations of low-frequency flexural and extensional vibrations of plates from the three-dimensional equations. They started with full expansions in infinite series of powers of the thickness-coordinate and then exercised great ingenuity in discarding higher powers and combining what was left so as to reach the desired equations. They had before them the ingredients of the higher order equations, but there was no interest in high-frequency vibrations at that time. Not long afterwards, Kirchhoff introduced energy considerations and integral theorems into the theory of plates; but he, too, was interested only in low frequencies and included just enough terms of the series for 
his immediate purpose. He did settle, though, the difficult question of boundary conditions.

In recent years there has been much interest in higher order theories of plates. Some of the developments have been based on analogy with the Bress-Timoshenko theory of high-frequency, flexural vibrations of bars, while others have been based on more fundamental energetic considerations. The earliest of the latter is E. Reissner's theory of flexure which appeared in 1945. Since that time there have been produced about as many ways of deriving higher order theories of bars, plates and shells as there have been authors to write about them. My own preference is to return to Poisson, Cauchy and Kirchhoff and pick up where they left off.

In Chapter 3 the three-dimensional equations of elasticity are converted to an infinite series of two-dimensional equations. This is done by expanding the displacement, in the variational equation of motion, in an infinite series of powers of the thickness-coordinate of the plate and integrating through the thickness. It seems to me that this is what Cauchy and Poisson might have done if they had had available what Kirchhoff was to develop later. The infinite series of equations are, of course, no easier to handle than the original equations; but the series-expressions of displacement, strain, stress, energy and equations of motion are of aid in deciding what to include in various orders of approximation and in understanding the implications of what is discarded and what retained.

The remaining three chapters deal with a variety of truncations of the series-expressions. In each case an explanation is given of how and why the truncation is performed. Comparisons are made between the resulting predictions of frequency vs. wave-length in an infinite plate and the corresponding relations obtained from the three-dimensional theory, where the latter are available. These comparisons serve to delineate the ranges of usefulness of the approximate equations. In many cases the estimates have been confirmed by comparison of particular solutions 
with experiments. The details are described in the papers listed in the Appendix.

The display of the infinite-series expansion, in Chapter 3, could lead the reader to expect that the subsequent process of truncation might be executed with some semblance of mathematical rigor. This is not the case. The series-expressions serve only to exhibit the branches which must be pruned. Where the cuts are made depends on physical considerations and on intuition based, in turn, on an understanding of the solution of the three-dimensional equations for the case of the infinite plate. However, when a truncation is completed, the kinetic energy-density and the strain-energy-density are formulated and a theorem of uniqueness of solutions of the approximate equations is established. Thus, each order of approximation is finally expressed as a self-sufficient mathematical system.

December 1955

R. D. Mindlin 
This page is intentionally left blank 


\section{Contents}

Foreword $\quad$ vii

Preface $x i$

Chapter 1: Elements of the Linear Theory of Elasticity 1

1.01 Notation 1

1.02 Principle of Conservation of Energy 6

1.03 Hooke's Law 7

$\begin{array}{ll}1.04 \text { Constants of Elasticity } & 10\end{array}$

1.05 Uniqueness of Solutions 15

$\begin{array}{ll}1.06 \text { Variational Equation of Motion } & 19\end{array}$

$\begin{array}{ll}1.07 \text { Displacement-Equations of Motion } & 20\end{array}$

Chapter 2: Solutions of the Three-Dimensional Equations 23

2.01 Introductory 23

2.02 Simple Thickness-Modes in an Infinite Plate 23

2.03 Simple Thickness-Modes in an Infinite, Isotropic Plate 25

2.04 Simple Thickness-Modes in an Infinite, Monoclinic Plate $\quad 29$

2.05 Simple Thickness-Modes in an Infinite, Triclinic Plate

2.06 Plane Strain in an Isotropic Body 34

$\begin{array}{ll}2.07 & \text { Equivoluminal Modes } \\ 2.08 & 35\end{array}$

2.08 Wave-Nature of Equivoluminal Modes 38 
2.09 Infinite, Isotropic Plate Held between Smooth, Rigid Surfaces (Plane Strain)

2.10 Infinite, Isotropic Plate Held between Smooth, Elastic Surfaces (Plane Strain)

2.11 Coupled Dilatational and Equivoluminal Modes in an Infinite, Isotropic Plate with Free Faces (Plane Strain)

2.12 Three-Dimensional, Coupled Dilatational and Equivoluminal Modes in an Infinite, Isotropic Plate with Free Faces

2.13 Solutions in Cylindrical Coordinates $\quad 75$

$\begin{array}{ll}2.14 & \text { Additional Boundaries } \\ \end{array}$

Chapter 3: Infinite Power Series of Two-Dimensional Equations 79 $\begin{array}{lll}3.01 & \text { Introductory } & 79\end{array}$

$\begin{array}{lll}3.02 & \text { Stress-Equations of Motion } & 81\end{array}$

$\begin{array}{lll}3.03 & \text { Strain } & 86\end{array}$

3.04 Stress-Strain Relations $\quad 90$

3.05 Strain-Energy and Kinetic Energy 91

3.06 Uniqueness of Solutions $\quad 94$

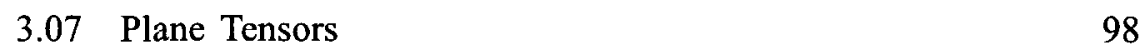

Chapter 4: Zero-Order Approximation 101

4.01 Separation of Zero-Order Terms from Series 101

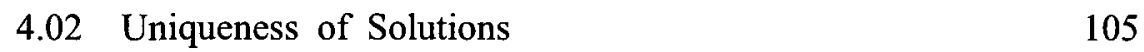

$\begin{array}{lll}4.03 & \text { Stress-Strain Relations } & 108\end{array}$

$\begin{array}{ll}4.04 & \text { Displacement-Equations of Motion } \\ 4.05 & 110\end{array}$

4.05 Useful Range of Zero-Order Approximation 112

Chapter 5: First-Order Approximation 115

5.01 Separation of Zero- and First-Order Terms from Series 
5.02 Adjustment of Upper Modes

5.03 Uniqueness of Solutions

5.04 Stress-Strain Relations

5.05 Stress-Displacement Relations

5.06 Displacement-Equations of Motion

5.07 Useful Range of First-Order Approximation

Chapter 6: Intermediate Approximations

6.01 Introductory

6.02 Thickness-Shear, Thickness-Flexure and Face-Extension

6.03 Thickness-Shear and Thickness-Flexure

6.04 Classical Theory of Low-Frequency Vibrations of Thin Plates

6.05 Moderately-High-Frequency Vibrations of Thin Plates

References

Appendix Applications of the First-Order Approximation

Biographical Sketch of R. D. Mindlin

Students of R. D. Mindlin

Presidential Medal for Merit

National Medal of Science 